\title{
Hematologic Disorders of COVID-19 and Appropriate Intensity of Exercise in Coronavirus Prevalence Period
}

\author{
Sara Zare Karizak ${ }^{1,{ }^{*}}$ and Majid Kashef ${ }^{2}$ \\ ${ }^{1}$ Sport Science Department, Faculty of Humanities, Persian Gulf University, Bushehr, Iran \\ ${ }^{2}$ Exercise Physiology Department, Sport Science Faculty, Shahid Rajaee Teacher Training University, Tehran, Iran \\ "Corresponding author: Sport Science Department, Faculty of Humanities, Persian Gulf University, Bushehr, Iran. Email: szk6699@gmail.com \\ Received 2021 January 24; Revised 2021 May 18; Accepted 2021 May 28.
}

Keywords: COVID-19, Hematopoiesis, Hemostasis, Exercise

\section{Dear editor,}

Coronavirus-associated disease (COVID-19) is a problem that has recently plagued human society worldwide (1). Fever, dry cough, and shortness of breath are the most important symptoms, and its complications range from upper respiratory tract infection to pneumonia and death (1). COVID-19 is a systemic infection that particularly affects the process of hematopoiesis and homeostasis (2). Coronavirus attacks blood cells, and in addition to increasing their apoptosis, during the autoimmune process by causing infection in the bone marrow, it also disrupts the production and differentiation of these cells and gradually reduces their amount (2). As a result, it reduces their function. Also, no basic treatment for this disease has been discovered, and the only way to avoid this problem is prevention. Therefore, it is necessary to know the factors affecting this disease and how to manage its complications. For this purpose, we reviewed studies of hematologic disorders in patients with COVID-19 and the same hematologic disorders caused by high intensity exercise. Studies have shown that the two proteins ORF3a and ORF10 in the coronavirus attack the heme part of the hemoglobin beta chain and are effective in increasing the lack of oxygen in hemoglobin and thus reducing the oxygen saturation of the blood (3). In this regard, Moradi et al. (4), in their case study, have shown a decrease in blood oxygen saturation to $63.4 \%$, which was associated with a decrease in red blood cells and hemoglobin in these patients. It has also been shown that following the reduction of oxygen saturation, deoxygenated hemoglobin will be more susceptible to virus attack (3); and a higher initial hemoglobin level is associated with a wider attack of coronavirus. This is one of the possible reasons for the higher prevalence in men compared to women due to their higher hemoglobin
(3). Simultaneously with this widespread attack and decrease in oxygenated hemoglobin, in addition to respiratory disorders, which is the most important complication of COVID-19, other side effects appear in the body subsequently. For example, disorders of iron metabolism and increased iron release from hemoglobin have been shown to cause oxidative stress and inflammation in the body (5). In addition, the coronavirus has been shown to cause systemic inflammation in the body by entering the epithelial cells and interacting with the receptor 2 of the angiotensin converting enzyme (ACE2), and many capillaries become susceptible to inflammation (6). As mentioned, cytokine storm and vascular injury or a combination of these two factors are the source of acute respiratory disorder syndrome (ARDS) as the most important complication of COVID-19 (6). These extensive capillary endothelial lesions are followed by increased coagulation reactions, platelet activation, increased fibrinogen and thrombosis in many body structures, especially in the lungs, and can lead to complications such as deep vein thrombosis (DVT), pulmonary embolism (PE), and disseminated intravascular coagulation (DIC) (7). It has been reported that more than $70 \%$ of patients with COVID-19 develop intravascular coagulation, and it has also been shown that there is a significant relationship between inflammatory factors and D-dimer peak values, which is an important coagulation marker (8). Qu et al. have associated higher platelet counts with more hospitalization days in these patients and reported a higher platelet-to-lymphocyte ratio (PLR) as an important inflammatory index in these patients (9). Connors and Levy also reported an increase in coagulation parameters such as platelet count, fibrinogen levels, and D-dimer in COVID-19 patients (10), while some studies have reported platelet depletion and coagulation factors in COVID-19 pa- 
tients. For example, Xu et al. have shown a decrease in circulating platelets (thrombocytopenia) and impaired coagulation in these patients (11). In addition, it has been suggested that the anemia and severe hemoglobin depletion due to coronavirus attack may be associated with systemic coagulation in patients with COVID-19 (3). Cases of hemolysis have also been reported with confirmation of decreased hemoglobin in the blood or increased hemoglobin in the urine (hemoglobinuria) in some COVID-19 case studies (12, 13). It has been shown that some factors, such as erythrocyte infection or their immune hemolysis exacerbate coronavirus attacks on the hemoglobin beta chain (3). In this regard, cases of autoimmune hemolytic anemia (AIHA) have been reported in COVID-19 patients $(14,15)$. In fact, immune hemolysis is a phenomenon in which the rupture of the erythrocyte membrane and the dissolution of its matrix occurs due to the reaction of antigen and antibody, during which red blood cells containing hemoglobin are removed from the cell and dissolve in plasma. Therefore, hemoglobin will not be able to carry oxygen (3). It has also been shown that high levels of blood lactate in COVID19 patients increase the risk of complications associated with the disease. For example, Nardi et al. examined the difference between arterial blood lactate and central venous blood levels in COVID-19 patients and reported an increase in this difference as a sign of impaired mitochondrial metabolism in the lung cells of these patients (16). Taneri et al. have also reported decreased hemoglobin and erythrocytes and increased ferritin in COVID-19 patients (17). Cavezzi et al. have also reported hemoglobinopathy, hypoxia, and iron overload in cells and tissues and increased toxic free iron in the blood circulation of these patients (5). On the other hand, studies have shown that various biological, chemical, and physical factors are effective in causing hematological disorders such as immune process disorders and increased coagulation and hemolysis. One of these influential factors is a high-intensity exercise (18). In this regard, studies have shown that the damage caused by frequent and severe muscle contraction or vasoconstriction in internal organs and factors such as hyperthermia, dehydration, hypoxia, shear stress due to high blood pressure, lactic acid, oxidative stress, proteolysis, and increasing concentration of catecholamines can be effective in increasing hemolysis, leukocytosis and coagulation processes due to high-intensity exercise (19-21). In addition, studies have shown that a high increase in lactic acid during strenuous exercise is effective in increasing glomerular permeability and thus renal excretion of red blood cells (hematuria) (22). Also, Mairbäurl, in their review article, have reported cases of hemolysis due to strenuous exercise (18). Whereas, Montero and Lundby, in their review article, have mentioned the increase of red blood cells and increase of plasma volume, and improvement of blood circulation characteristics due to adaptation with moderate-intensity training (23). Van der Vorm et al., in their review article, reported hemostatic disorders and platelet aggregation and vice versa, the effect of moderateintensity training sessions in reducing the factors affecting clot formation and platelet aggregation (24). Jamurtas et al. also showed disorders in white blood cells and redox status after high intensity training compared to moderate aerobic training (20). Therefore, due to the importance of high-intensity exercise in causing temporary disorders of blood components such as leukocytes, erythrocytes, hemoglobin, and platelets and on the other hand, effects of COVID-19 on these disorders such as immunosuppression, an increase of hemolysis and coagulation. It seems necessary to manage the current situation and prevent the risk of infection with coronavirus and its symptoms. Therefore, it is recommended that ordinary people refrain from high-intensity exercise until the disease is uncontrolled and doing moderate-intensity exercise is a safer approach for them during this period. Also, because high-intensity exercise is inevitable for professional athletes, firstly, it is recommended to suspend professional exercise or competitions until the disease is uncontrolled. Secondly, it is recommended to consider enough time after high-intensity exercise for recovery. Since studies have shown hematologic disorders sometimes remain up to 72 hours after high-intensity exercise (25), therefore it is recommended to consider at least 72 hours after highintensity exercise to ensure the entire recovery. Also, another recommendation for professional athletes is doing interval exercise sessions with high intensity and moderate intensity between them in the week to ensure proper recovery and prevention of hematologic disorders in the coronavirus prevalence period.

\section{Footnotes}

Authors' Contribution: Both authors have been involved in gathering information, writing, and reviewing the article.

Conflict of Interests: There was no conflict of interest. Funding/Support: There has been no funding/support.

\section{References}

1. Hassanzadeh-Rad A, Halabchi F. Stadiums as possible hot spots for COVID-19 spread. Asian J Sports Med. 2020;11(2). doi: 10.5812/asjsm.103178.

2. Karimi Shahri M, Niazkar HR, Rad F. COVID-19 and hematology findings based on the current evidences: A puzzle with many missing pieces. Int J Lab Hematol. 2021;43(2):160-8. doi: 10.1111/ijlh.13412. [PubMed: 33264492]. [PubMed Central: PMC7753300]. 
3. Liu W, Li H. COVID-19:Attacks the 1-beta chain of hemoglobin and captures the porphyrin to inhibit human heme metabolism. ChemRxiv. 2020;Preprint. doi: 10.26434/chemrxiv.11938173.v9.

4. Moradi M, Yekefallah L, Sedighi M, Ghapanvari F, Namdar P. [Delayed pneumomediastinum and subcutaneous emphysema in Covid-19 patients: A case report]. J Qazvin Univ Med Sci. 2020;24(2):190-201. Persian. doi: 10.32598/jqums.24.2.433.8.

5. Cavezzi A, Troiani E, Corrao S. COVID-19: Hemoglobin, iron, and hypoxia beyond inflammation. A narrative review. Clin Pract. 2020;10(2):1271. doi: 10.4081/cp.2020.1271. [PubMed: 32509258] [PubMed Central: PMC7267810].

6. Amraei R, Rahimi N. COVID-19, renin-angiotensin system and endothelial dysfunction. Cells. 2020;9(7). doi: 10.3390/cells9071652. [PubMed: 32660065]. [PubMed Central: PMC7407648].

7. Eljilany I, Elzouki AN. D-dimer, fibrinogen, and IL-6 in COVID-19 patients with suspected venous thromboembolism: A narrative review. Vasc Health Risk Manag. 2020;16:455-62. doi: 10.2147/VHRM.S280962. [PubMed: 33223833]. [PubMed Central: PMC7672709].

8. Al-Samkari H, Karp Leaf RS, Dzik WH, Carlson JCT, Fogerty AE, Waheed A, et al. COVID-19 and coagulation: Bleeding and thrombotic manifestations of SARS-CoV-2 infection. Blood. 2020;136(4):489-500. doi: 10.1182/blood.2020006520. [PubMed: 32492712]. [PubMed Central: PMC7378457].

9. Qu R, Ling Y, Zhang YH, Wei LY, Chen X, Li XM, et al. Plateletto-lymphocyte ratio is associated with prognosis in patients with Coronavirus disease-19. I Med Virol. 2020;92(9):1533-41. doi: 10.1002/jmv.25767. [PubMed: 32181903]. [PubMed Central: PMC7228291].

10. Connors JM, Levy JH. COVID-19 and its implications for thrombosis and anticoagulation. Blood. 2020;135(23):2033-40. doi 10.1182/blood.2020006000. [PubMed: 32339221]. [PubMed Central: PMC7273827].

11. Xu P, Zhou Q, Xu J. Mechanism of thrombocytopenia in COVID-19 patients. Ann Hematol. 2020;99(6):1205-8. doi: 10.1007/s00277-02004019-0. [PubMed: 32296910]. [PubMed Central: PMC7156897].

12. Verdiyeva N, Ibrahimova T, Nasibova A, Huseynov V. How to treat and manage COVID19 in SCD patients. Hematol. 2020;42:76. doi: 10.1016/j.htct.2020.09.137.

13. De Franceschi L, Costa E, Dima F, Morandi M, Olivieri O. Acute hemolysis by hydroxycloroquine was observed in G6PD-deficient patient with severe COVD-19 related lung injury. Eur J Intern Med. 2020;77:1367. doi: 10.1016/j.ejim.2020.04.020. [PubMed: 32381323]. [PubMed Central: PMC7167571].

14. Sahu KK, Borogovac A, Cerny J. COVID-19 related immune hemolysis and thrombocytopenia. J Med Virol. 2021;93(2):1164-70. doi: 10.1002/jmv.26402. [PubMed: 32776538]. [PubMed Central:
PMC7436763].

15. Wahlster L, Weichert-Leahey N, Trissal M, Grace RF, Sankaran VG. COVID-19 presenting with autoimmune hemolytic anemia in the setting of underlying immune dysregulation. Pediatr Blood Cancer. 2020;67(9). e28382. doi: 10.1002/pbc.28382. [PubMed: 32495391]. [PubMed Central: PMC7674227].

16. Nardi G, Sanson G, Tassinari L, Guiotto G, Potalivo A, Montomoli J, et al. Lactate arterial-central venous gradient among COVID-19 patients in ICU: A potential tool in the clinical practice. Crit Care Res Pract. 2020;2020:4743904. doi: 10.1155/2020/4743904. [PubMed: 33014462]. [PubMed Central: PMC7519437].

17. Taneri PE, Gomez-Ochoa SA, Llanaj E, Raguindin PF, Rojas LZ, RoaDiaz ZM, et al. Anemia and iron metabolism in COVID-19: A systematic review and meta-analysis. Eur J Epidemiol. 2020;35(8):763-73. doi: 10.1007/s10654-020-00678-5. [PubMed: 32816244]. [PubMed Central: PMC7438401].

18. Mairbaurl H. Red blood cells in sports: effects of exercise and training on oxygen supply by red blood cells. Front Physiol. 2013;4:332. doi: 10.3389/fphys.2013.00332. [PubMed: 24273518]. [PubMed Central: PMC3824146]

19. Lippi G, Sanchis-Gomar F. Epidemiological, biological and clinical update on exercise-induced hemolysis. Ann Transl Med. 2019;7(12):270. doi: 10.21037/atm.2019.05.41. [PubMed: 31355237]. [PubMed Central: PMC6614330].

20. Jamurtas AZ, Fatouros IG, Deli CK, Georgakouli K, Poulios A, Draganidis D, et al. The effects of acute low-volume HIIT and aerobic exercise on leukocyte count and redox status. J Sports Sci Med. 2018;17(3):501-8. [PubMed: 30116124]. [PubMed Central: PMC6090390].

21. Ahmadizad S, Malekian E, Khani E, Rahmani H. Exercise and training effects on platelet activation and function: A review article. Sport Physiology (Research on Sport Science). 2019;11(43):17-38.

22. Bellinghieri G, Savica V, Santoro D. Renal alterations during exercise. J Ren Nutr. 2008;18(1):158-64. doi: 10.1053/j.jrn.2007.10.031. [PubMed: 18089464].

23. Montero D, Lundby C. Regulation of Red Blood Cell Volume with Exercise Training. Compr Physiol. 2018;9(1):149-64. doi: 10.1002/cphy.c180004. [PubMed:30549016].

24. van der Vorm LN, Huskens D, Kicken CH, Remijn JA, Roest M, de Laat B, et al. Effects of repeated bouts of exercise on the hemostatic system. Semin Thromb Hemost. 2018;44(8):710-22. doi: 10.1055/s-0038-1673619. [PubMed: 30290394].

25. Galazka-Franta A, Jura-Szoltys E, Smolka W, Gawlik R. Upper respiratory tract diseases in athletes in different sports disciplines. $J$ Hum Kinet. 2016;53:99-106. doi: 10.1515/hukin-2016-0014. [PubMed: 28149415]. [PubMed Central: PMC5260580]. 